\title{
SMART POLYMER NANOCOMPOSITES: RECENT ADVANCES AND PERSPECTIVES
}

\author{
MYLEIDI VERA, CLAUDIO MELLA AND BRUNO F. URBANO * \\ Departamento de Polímeros, Facultad de Ciencias Químicas, Universidad de Concepción, Chile.
}

\begin{abstract}
Nanocomposite polymers have received considerable interest in research for the last three decades. Those nanocomposite polymers that are sensitive to a stimulus such as $\mathrm{pH}$, temperature, magnetism, and electricity, among others, called smart or intelligent nanocomposite polymers had received even greater attention due to their potential technological applications. Applications of these polymers include flexible electronic devices, sensors, self-healing polymers, shape-memory materials, etc. The sensitivity of the material can come from both the polymer that acts as a matrix and the nanofiller, resulting in a material that combines properties of each of its components and that each one will not have separately. This mini-review aims to provide an update on the most recent and significant applications in the area of stimuli-responsive polymer nanocomposites, emphasizing the most innovative applications in biomedicine and catalysis developed in the last three years.
\end{abstract}

Keywords: Polymer nanocomposites, external stimuli, smart polymers, nanofillers, physical stimuli.

\section{INTRODUCTION}

Polymer nanocomposites (PNCs) are filler-reinforced polymers; in these materials, the filler size is characterized by being of the order of nanometers $(<100 \mathrm{~nm})$ in at least one dimension. Nanofillers can be classified in zerodimensional (spherical), one-dimensional (layered), and twodimensional (fibrous and tubular), according to the number of dimensions that are outside the nanometric range[1].

Since its inception, around the 90s[2], PNCs have emerged as materials of great interest in both academia and industry, because they are considered materials with superior properties in terms of cost, barrier resistance, thermal stability, lightweight, as well as optical, mechanical, and electrical properties[3]. Organic or inorganic nanofillers improve the already existing properties of polymers and introduce new properties to the hybrid material, expanding the possible applications, and preserving the ease of manufacture and processing characteristic of polymers[4]. The unique properties of PNCs can be attributed to the nanosize of the filler. Different from micro or macroscopic fillers, nanofillers provide an extremely high surface-tovolume ratio, increasing fillermatrix, and filler-filler interactions[5]. These unique properties have provided these materials with multiple commercial successes, including flame retardants, stimulus-responsive materials, and automotive parts[6].

In recent years, efforts have been made to integrate the properties of polymers and nanofillers in the creation of materials with stimulus-response properties. Consequently, a large number of organic and inorganic materials have been used as passive and active nanofillers. Active nanofillers are usually responsible for amplifying or generating a nanocomposite material response to any stimulus (chemical or physical)[5].

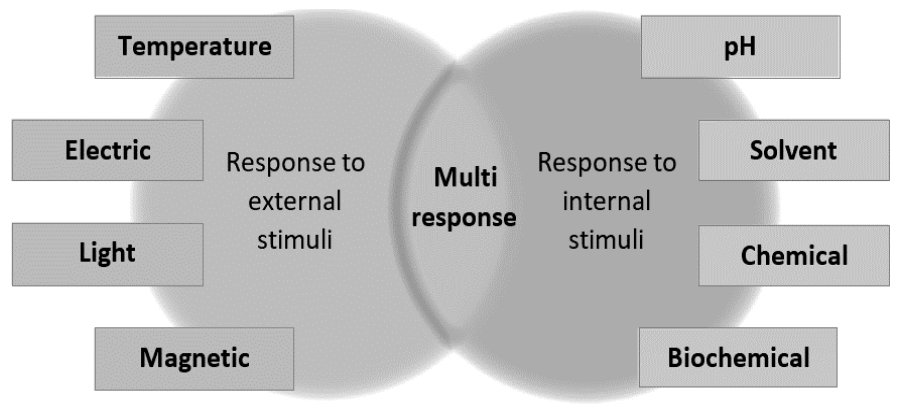

Figure 1. Classification of the responsive nanocomposite.

Smart or intelligent materials are characterized by having one or more physical and chemical properties that can be controlled or changed by the action of a stimulus[7, 8]. This stimuliresponsive characteristic can be classified according to this nature as internal or chemical stimuli, and external or physical stimuli. The chemical stimuli correspond to $\mathrm{pH}$, solvent, chemical recognition, and biological or enzymatic recognition. On the other hand, the physical stimuli correspond to temperature, electric current, light, and magnetic field (see Figure 1)[5]. Physical stimuli are very appreciated due to is possible to establish and control the parameter easily than chemical stimuli, opening a large opportunity to apply them on electrotechnological solutions, supported by advances in medicine and 3D printing technology. Multistimuli-responsive nanocomposites can react to one or more stimuli, and their properties depend on the characteristics of the polymer matrix and the type of nanofiller used[9]. In recent years, there has been a notable increase in the use of multistimuliresponsive PNCs for various types of biomedical[10], automotive[11], aerospace[12], and biotechnological applications[13], which is reflected in the rise in the number of publications in the last ten years (see Figure 2).

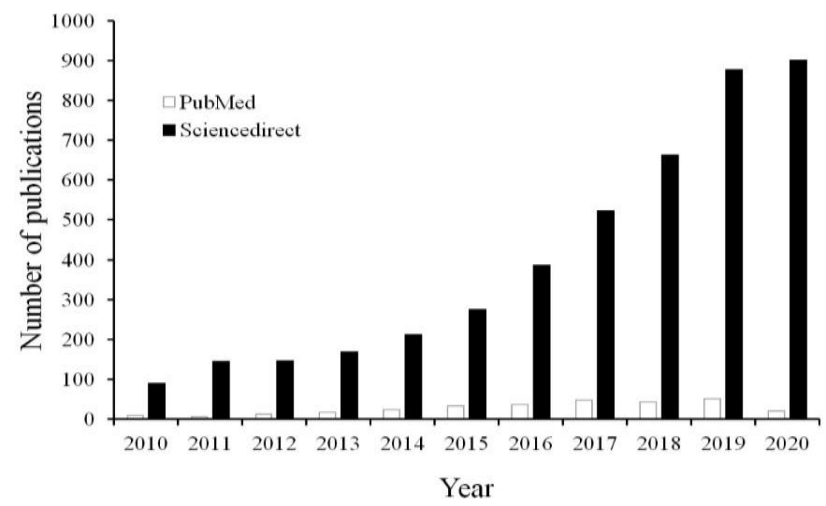

Figure 2. A number of publications per year of the term "stimuli polymer nanocomposites" for the period 2010-2020. Data from ScienceDirect and PubMed on July 23, 2020.

This mini-review aims to provide an update on the most recent and significant applications in stimuli-responsive PNCs. This work is divided into two sections. The first gives an overview of PNCs. The second section focuses on the different stimuli-responsive (light, magnetic field, electric field, and temperature) polymer nanocomposite emphasizing the most recent biomedical and catalytic applications developed in the last three years.

\section{POLYMER NANOCOMPOSITES}

Composite materials are formed by the union of two or more materials with different chemical or physical properties. These materials are characterized by keeping separated within the same structure. The constituent materials of composites are classified into two categories: matrix and reinforcement. Matrix materials are responsible for maintaining the positions of the reinforcement materials, by surrounding and supporting them. Reinforcement materials contribute to their chemical or physical properties, providing new properties to the matrix. As a result, composite materials possess combined properties of both materials (matrix and reinforcement). However, the chemical and physical properties of composites differ entirely from the materials that compose it $[8,14]$. 
Polymers have proven to be materials with exceptional properties such as ease of production, low cost, high flexibility, biocompatibility, excellent machinability, and lightweight, to name a few. However, its use is sometimes limited by its low modules, low resistance, and low conductivity compared to materials such as metals or ceramics. One alternative to improve these properties is by inserting small amounts of nanofillers of different shapes and nature. This reinforcement has been proven for years to enhance mechanical, chemical, thermal, optical, and electrical properties, as well as to provide the ability to respond to stimuli[15-17].

A wide variety of organic and inorganic nanofillers have been combined with different types of polymers to obtain PNCs designed with specific properties for each type of application. Organic nanofillers include polymer nanofiber, natural fibers (cellulose, flax, wood, etc.), and natural clay[18, 19]. Among inorganic nanofillers are nanoclays, metal oxides $\left(\mathrm{Cu}_{2} \mathrm{O}, \mathrm{MgO}, \mathrm{Fe}_{2} \mathrm{O}_{3}\right.$, etc.), carbon nanofillers (graphene, carbon fibers, graphite, etc.), metallic particles ( $\mathrm{Au}, \mathrm{Ag}$, $\mathrm{Fe}$, etc.), and other particles ( $\mathrm{PbS}, \mathrm{CdS}, \mathrm{MoS}_{2}$, etc.)[20-23].

Thanks to the development of nanotechnology, a great interest has been generated in the development of nanocomposite materials. The integration of different nanofillers to polymeric matrices has been shown to improve mechanical properties and opens up the possibility of creating new materials with a capacity to respond to different chemical, physical, or biological stimuli[24]. Different types of nanofillers have been used to obtain nanocomposites, which can be subdivided into two categories according to their ability to respond to stimuli: i) inert nanofillers, which lack stimulus-responsive properties and their primary role is the mechanical reinforcement. These nanofillers include silica, cellulose, and clay materials nanoparticles (montmorillonite, hectorite, saponite, laponite, halloysite, etc.)[25-27]. ii) Active nanofillers, this type of nanofillers has stimulus-responsive properties, which in addition to reinforcement, provides the stimulus-response capacity to the nanocomposite material.
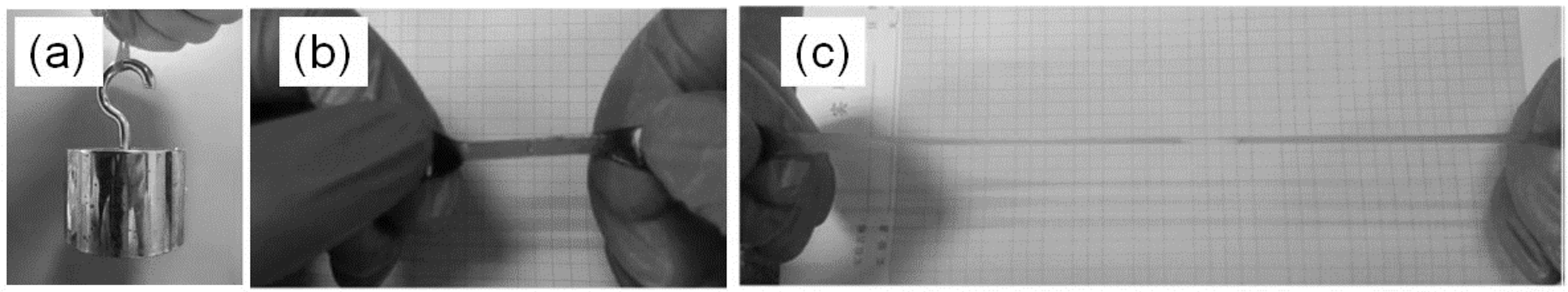

Figure 3. PNIPAM/MXene hydrogel showing high strength (a), self-healed PNIPAM/MXene hydrogel (b), and self-healed PNIPAM/MXne hydrogel being stretched (c). (Adapted with permission from[34]. Copyright (2019) American Chemical Society).

Another aspect that significantly influences the properties of nanocomposites is the shape of the nanofiller, as it substantially affects the percolation threshold. Among the different nanofiller shapes are nanotubes, nanofibers, nanorods, nanoparticles, and nanowires[35-37]. The sheets are among the least used fillers for developing stimulus-response materials, although they have great potential[38]. Conversely, spherical and rod-type fillers are the most widely used for this type of application[5].

The nanofillers in nanocomposites have very large interfacial areas due to their small size. The interfacial region is defined as the interface region where the properties differ from those of the bulk. This region can vary in size ranging from 2 to $50 \mathrm{~nm}$ and is characterized by alterations in chemical properties, polymer mobility, degree of cure, and crystallinity. The degree of interaction between the filler and the polymer matrix are critical in determining the properties of the PNCs[39]

Nanofillers generally interact with the matrix through weak intermolecular forces. However, in some cases, the interaction occurs through chemical bonds. One of the most relevant aspects of the synthesis of PNCs is the good dispersion of the filler inside the matrix. In general, the good dispersion of nanofillers in a polymer matrix is difficult since the nanoparticles have high surface energy, which favors their agglomeration[40]. Additionally, the incorporation of inorganic nanoparticles is limited by the incompatibility between hydrophilic nanoparticles and polymers with hydrophobic chains, resulting in poor surface interaction. Two strategies have been addressed to solve these drawbacks: the use of new synthesis methods and the superficial modification of the nanofillers[40].
Examples of this type of material include carbon-based, gold, and magnetic iron oxide nanofillers $[28,29]$.

For the synthesis of stimuli-response nanocomposites, active nanofillers are mostly used because these materials can react to different stimuli. As a result, the obtained nanocomposite could respond to electric currents, light, temperature, magnetic field, $\mathrm{pH}$ changes, etc[30-32]. An example that demonstrates the effect that active nanofillers can generate when incorporated into polymer matrices is the work carried out by Chen et al., who reported the synthesis of a shapememory nanocomposite with a rapid light response and self-healing performance. The matrix of the nanocomposite was composed of the mixture of poly( $\varepsilon$-caprolactone)/thermoplastic polyurethane. Polydopamine nanospheres were incorporated as nanofiller, which gave the system a memory response shape effect to light with a shape recovery ratio of $100 \%$. Additionally, the PNCs showed excellent self-healing performance in response to irradiation with light for $150 \mathrm{~s}$. The resulting material also exhibited excellent mechanical properties (tensile strength 1.6 MPa), making it ideal for biomedical applications[33] Similarly, in the work carried out by Zhang, et al., the synthesis of a nanocomposite polymer composed of MXene and poly ( $N$-isopropyl acrylamide) (PNIPAM) hydrogel was reported. MXene is a twodimensional crystalline material with excellent mechanical properties, electrical conductivity, and thermal conductivity. PNIPAM is a thermosensitive polymer widely used as a matrix material for temperature sensing. The resulting nanocomposite material exhibited excellent mechanical properties: it could be stretched to over 14 times the original length and achieved a 0.4 MPa tensile strength while showing good self-healing ability (see Figure 3). Additionally, due to the incorporation of the nanofiller, the nanocomposite hydrogel showed a conductivity of $1,092 \mathrm{~S} / \mathrm{m}$, approximately 15 times that of the control hydrogel without MXene. The material obtained showed excellent properties to be used as an intelligent compression sensor[34].

\subsection{Synthesis methods}

The synthesis of PNCs is a challenging process that has been approached from different strategies, currently finding a variety of processing techniques. These techniques include in situ polymerization, melt compounding, and solution mixing[41, 42]. In in-situ polymerization, the nanofillers and the monomer solution are mixed at the beginning of the reaction. The dispersion of the nanofillers is carried out as the polymerization occurs, although in some cases, the reaction rate is affected by the presence of the filler[41]. In melt compounding, the nanofiller mixes with the molten polymer, reducing the need for solvent, although the reaction is generally carried out at high temperatures[43]. Finally, in the solution mixing, a dispersed solution of nanofillers is mixed with the polymer solution. Once the mixture is homogenized, the solvent is evaporated to obtain the nanofillers dispersed in the polymer. This technique is easy to perform and requires low temperatures. However, the solvent must be carefully selected as it must be capable of dispersing the nanofillers well and dissolving the polymer[42].

Although the development of a universal method for the synthesis of nanocomposite polymers is difficult due to the characteristics of the polymer matrix, the nanofiller, as well as their physical and chemical interactions, the synthesis methods described above have shown excellent results, even for systems with nanofillers and polymers of different nature. Wu et al., reported the synthesis of $\mathrm{Pd}, \mathrm{Pt}, \mathrm{Cu}$, and $\mathrm{Au} /$ poly(methyl methacrylate) nanocomposites, obtained by the in situ polymerization method. As a result, they obtained systems with uniform distribution of nanofillers in the polymer matrix. 
Additionally, they reported improvements in tensile strength compared to pure polymer for almost all of the systems studied[44]. Herrero M. et al., reported the synthesis of bio-based polyamide 11/sepiolite nanocomposites using melt compounding and in situ polymerization. As a result, in both synthesis methods, the sepiolite dispersed homogeneously within the polymer matrix, and improvements in Young's modulus, tensile strength, and thermal distortion temperature were reported; however, in situ polymerization allowed to achieved better dispersions of the clay[45]. Finally, Bayraktar et al., reported the synthesis of silver nanowire/polylactide nanocomposites using the solution mixing method and its subsequent 3D printed to obtain desired shapes. The obtained material showed an excellent dispersion of the nanofiller in the matrix and excellent antibacterial properties. This work demonstrated that the solution mixing method turns out to be efficient for use in 3D printing, combining the versatility of polymers for $3 \mathrm{D}$ printing and the antibacterial properties of some nanofillers[46].

In some cases, such as for clay nanocomposites, the synthesis method's specific choice depends on the morphology required for the nanocomposite, that is, exfoliated or intercalated[47]. In intercalated morphology, the polymer chains are inserted between the ordered layers of the clays. While the exfoliated morphology, the clay layers are separated and distributed within the matrix. In this case, the exfoliation capacity will depend on the nature of the clay, the agents used for curing, and the type of mixture[48]. These arrangements are usually obtained by melt compounding or by in situ polymerization. According to the synthesis process carried out, it is possible to obtain both types of configurations. Behniafar et al., reported montmorillonite platelets intercalated by using aminetelechelic poly(tetramethylene oxide) PTMO trimethylated to quaternary ammonium (QA)-capped PTMO. Subsequently, segmented polyurethanes were synthesized in situ to obtain exfoliated clay nanocomposites finally. According to the results obtained through the procedure carried out, it was possible to achieve full exfoliation of the clay platelets. Furthermore, the obtained nanocomposites polymers presented better storage moduli compared to polymer matrices in the absence of nanofillers[49]. However, it is also possible to achieve both structures in the same material. As reported by Wadi et al., they synthesized a nanocomposite composed of molybdenum disulfide $\left(\mathrm{MoS}_{2}\right)$ and isotactic polypropylene (iPP) using a one-step melt extrusion method. The material obtained presented both exfoliated and intercalated morphology. Additionally, mechanical tests revealed a significant increase in toughness and elongation at break (300-400 \%) in compounds containing low amounts of $\mathrm{MoS}_{2}(0.25$ to $0.5 \%$ by weight). Besides, the addition of a small quantity of $\mathrm{MoS}_{2}$ also improved the thermal stability of the polymer. The authors suggest that the bulk $\mathrm{MoS}_{2}$ and onestep melt extrusion process could be a scalable and straightforward method to induce high elongation and toughness in iPP[50].

\subsection{Surface modification of nanofillers}

The surface modification of nanofillers consists of introducing organic coatings through physical or chemical interactions, most often to enhance the compatibility between the matrix and the filler. In physical methods, nanofillers are usually covered by high molecular weight surfactants or polymers[51, 52]. In the case of chemical methods, the modification of the surface is carried out employing a covalent attachment of the modifier. The chemical interaction is more efficient to avoid desorption of the nanofiller and also gives the possibility of creating chemical bonds between the nanofiller and the polymer matrix[40]. These methods of surface modification of nanofiller have also shown to be useful for altering interfacial states and improving the physical and chemical properties of PCNs. In the work carried out by Cha et al., two nanocomposite systems in which an epoxy matrix was used were compared; the first system has carbon nanotubes and graphene nanoplatelets functionalized with melamine, and the second system has non-functionalized nanofillers. The results showed that nanocomposites with melaminefunctionalized nanofillers presented higher tensile strength and fracture resistance than nanocomposites that had nonfunctionalized nanofillers[53]. Similarly, in the work reported by Min et al., on nanocomposite polyimide using amine-functionalized graphene nanosheets, an increase in thermal stability, mechanical properties, and tribological performance was evidenced, when compared to non-functionalized graphene nanosheets nanocomposites[54].

\section{STIMULI-RESPONSIVE POLYMER NANOCOMPOSITE}

A responsive nanocomposite material is generally designed with the target to spontaneously respond through an alteration on the structure of softness material or moieties, based on synergistic integration of stimuli-responsive characteristics of both organic and inorganic components. Next, the application of physical stimuli and its some recent applications as catalytic materials, and especially in medical treatments and 4D printing, based on stimulus-sensitive 3D materials that modify its shape, will be reviewed.

\subsection{Temperature}

Thermoresponsive nanocomposites are usually employed as smart materials due to the temperature is the easiest external stimulus to apply. The thermoresponsive matrix undergoes a change in the structure associated with hydrogen bonds. Hydrogels are the most representative thermoresponsive polymers, which exhibit a reversible volume phase transition from the hydrolyzed state to a precipitated state of chains of polymers, with a significant volume change in response to temperature variation (see Figure 4). In the case of this transition phase of the polymer occurs when temperature decreases as occur in poly(acrylamide) (PAAm) and poly(acrylic acid) (PAA) copolymers, the transition temperature is called upper critical solution temperature (UCST) $[55,56]$. In contrast, if transition phase occurs with an increase in temperature, it is called lower critical solution temperature (LCST) such as the behavior of poly $(N$-vinyl caprolactam) (PVCL), $\operatorname{poly}(N, N$-dimethyl acrylamide) (PDEAAm), poly oxazoline (POx), poly[2-(dimethylamino)ethyl methacrylate] (PDMAEMA), block copolymers of polyethylene oxide (PEO), and poly $(N$ isopropyl acrylamide) (PNIPAAm)[57]. In particular, PNIPAAm is recognized in biomedical applications such as gene and drug delivery systems, due to its high biocompatibility and an LCST close to the human body temperature, but it present typical drawbacks like fragility and poor mechanical properties.

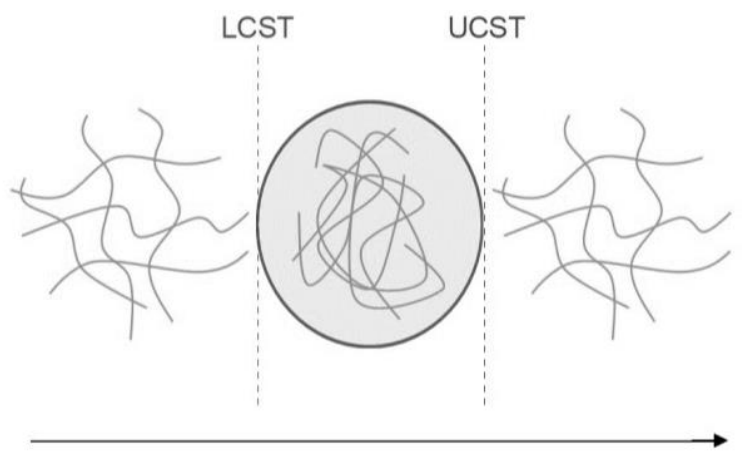

\section{Temperature}

Figure 4. Reversible phase transition of thermoresponsive polymers.

Besides the thermo-responsiveness of the matrix, it is possible to add nanofillers to improve the mechanical and drug release properties[58], and broaden its applications as substitutes for human tissues, artificial actuators, and cellular substrates. Different types of PNCs has been developed as bone substitutes. Nistor et al., designed a collagen/PNIPAAm hydroxyapatite nanocomposite. They have studied the nature of commercial hydroxyapatite, crosslinker degree of PNIPAAm hydrogel, and collagen composition. The authors considered the recognized strength and hemostatic properties of collagen fibers, osteoconductivity, and re-absorbable hydroxyapatite properties and the properties of PNIPAAm as artificial extracellular matrix above $33{ }^{\circ} \mathrm{C}$, can provide a good result as a replacement for bone tissue[59]. Oguz et al., designed a nanocomposite based on methylcellulose-gelatin hydrogels and different types of calcium phosphate fillers. The inorganic filler's content is related to an increment on the transition temperature of the materials from $25^{\circ} \mathrm{C}$ to $37^{\circ} \mathrm{C}$, which allows the use as injectable nanocomposite, which changes to a solid-state when entering on human bone tissue[60].

Other exciting applications is related to combination of the thermoresponsive of polymer behavior with the bactericidal effect of some metal nanoparticles. Epidermal burn damage provides a productive environment for microbial growth, making treating skin burns a difficult medical issue. Rapid bacterial growth is considered a challenge to prevent the development of resistance bacterias and makes the drug release process less effective. Arafa et al. report the design of Pluronic 127-gold particle nanocomposite to apply as a gold nanoparticle release system tested on skin burns of in vivo samples. 
The great advantage to synthesize well distribute nanoparticles at the hydrated state of hydrogel to then release at collapse form around $37{ }^{\circ} \mathrm{C}$, together with great bioadhesive behavior of hydrogel, provides excellent results as bactericidal treatment[61].

On the other hand, in chemistry technology, it is possible to synthesize switchable catalyst materials using thermoresponsive materials with noble metal nanoparticles or metal oxide with catalytic properties. Li et al. reported core Ag$\mathrm{Au}$ bimetallic nanoparticles covered by poly $(\mathrm{N}$-isopropyl acrylamide-co-3methacryloxypropyl trimethoxysilane) for 4-nitrophenol reduction. The catalyst present different behavior has shown a switch off catalytic properties near LCST due to a contraction of hydrogel chains over catalytic nanoparticles avoiding the access of nitrophenol reactant to the metallic surface[62]. A similar result was obtained by Feng et al., which prepared $\mathrm{ZnO}$ nanoparticles on the PNIPAAm matrix. The PNC showed a minor catalytic activity than pure $\mathrm{ZnO}$, but it was able to avoid the rapid degradation of oxide nanoparticle at $25^{\circ} \mathrm{C}$; furthermore, the catalytic activity is switch-off at $40{ }^{\circ} \mathrm{C}$ with negligible degradation of $\mathrm{ZnO}[63]$.

\subsection{Magnetic field.}

The magnetic nanoparticle is affected by static or alternating magnetic fields, and advantageously this magnetics field has a relatively large penetration depth and non-contact stimulation source. The magnetic response is produced due to the interaction of magnetic moments in material with the magnetic gradient produced by the magnetic field[64]. The most commonly used magnetic nanoparticles are metals, alloys, and metal oxides, where stand out the magnetite $\left(\mathrm{Fe}_{3} \mathrm{O}_{4}\right)$, due to it is easily synthesized and has superparamagnetic properties and great biocompatibility[65]. On this matter, polymers are considered as valuable materials for acting as a matrix to support and stabilize well-dispersed nanomagnetic particles to obtain a magnetic nanocomposite. The magnetic response PNC has been observed as a change in shape or movement of the nanocomposite. It can be used as magnetically separable materials for focalized drug delivery and purification systems. Furthermore, a thermal expression (or hyperthermia process) can be observed on magnetic materials in nanoscale. It has the property to be affected by alternating magnetic fields (AMF) to release heat by the oscillation of nanomagnetic filler, associate with Brownian and Néel relaxation process[64]. This property can be applied to artificial muscles (actuators materials), shape-memory polymers (SMP) or shape memory composites (SMC)[66], materials to kills cancer cells in tumors, or as nanocarriers for drug release.

Among different polymer matrix used for synthesis of SMP materials, the thermoplastic polyurethane (TPU) polymers stand out. Soto et al., synthesized a nanocomposite based on a commercial polyurethane and $\mathrm{Fe}_{3} \mathrm{O}_{4}$ nanoparticle using the casting and drying technique[67]. The effect of magnetite concentration $\left(1,7\right.$, and $\left.10 \% \mathrm{Fe}_{3} \mathrm{O}_{4}\right)$ in heat release and shape recovery properties when they were exposed to $\mathrm{AMF}$ were evaluated, finding a direct correlation between time to shape recovery with $\mathrm{Fe}_{3} \mathrm{O}_{4}$ concentration.

SMC also can be applied as a tissue scaffold or in medical application with a correct choice of the polymer matrix. Recently, Zhao et al., proposed a biocompatible nanocomposite based on $\mathrm{Fe}_{3} \mathrm{O}_{4}$ and polylactic acid (PLA) for 4D printing of a tracheal scaffold[68]. The mechanical and thermal characterization verify high toughness without loss of rigidity. Meanwhile, the application of 30 $\mathrm{kHz}$ AMF produces a rapid nanocomposite shape recovery from a square sheet shape to a cylindrical shape related to tracheal bio-shape. A similar material composition was proposed by Wei et al. with a $3 \mathrm{D}$ ink based on PLA, $\mathrm{Fe}_{3} \mathrm{O}_{4}$ and benzophenone as polymer matrix, nanofiller and UV initiator respectively. The nanocomposite is immediately crosslinked in 3D printing process[69]. A helicalshape with a controllable dimension and memory recovery ability, makes it a candidate material for application as an intravascular stent operable by external magnetic stimuli (see Figure 5).
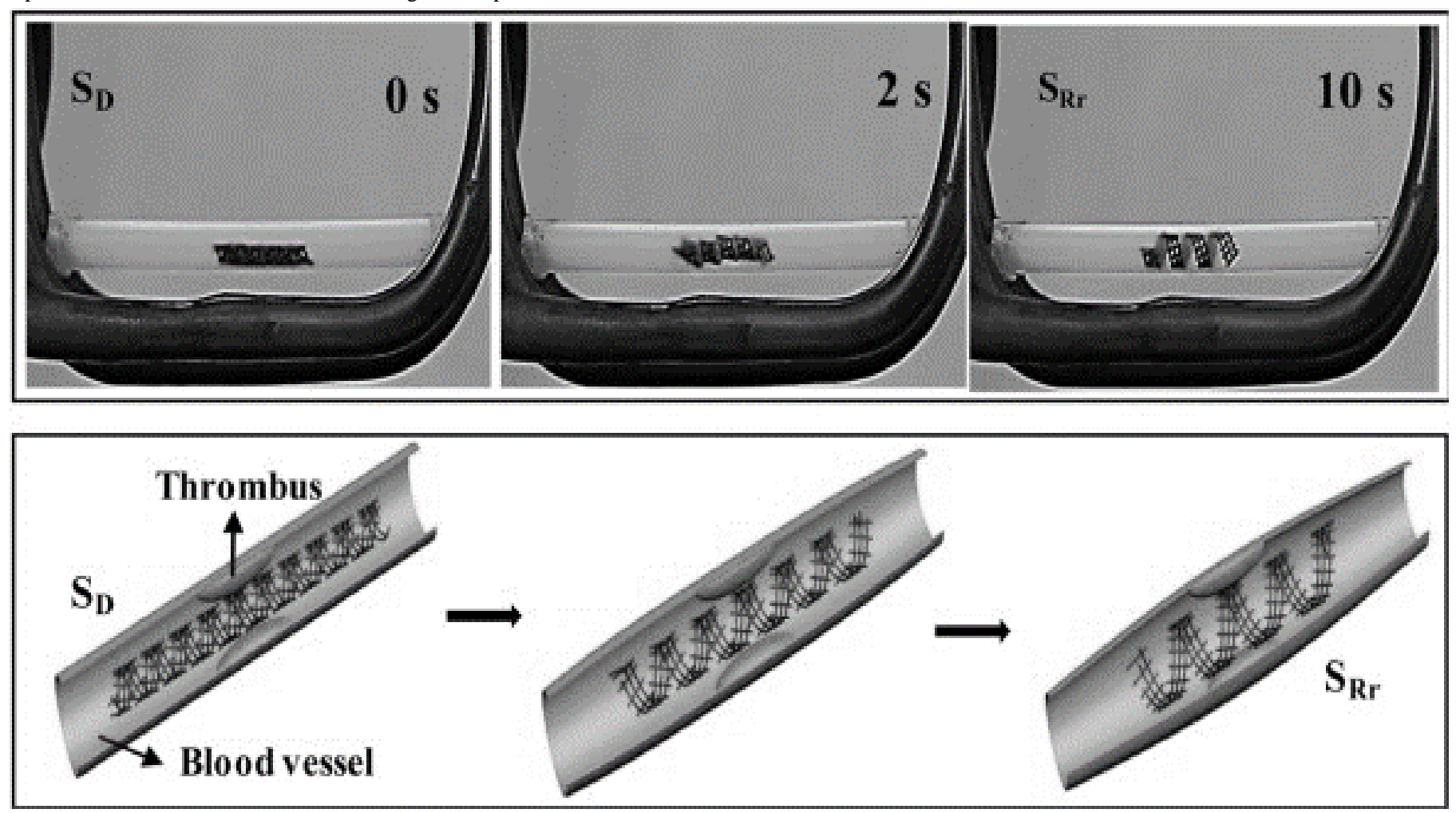

Figure 5. Helicoidal recovery shape design and potential application of 4D composite as an intravascular stent. (Adapted with permission of [69]. Copyright (2017) American Chemical Society)

In the nanocarrier and drug release area, Wang et al., proposed a magnetic chitosan nanocomposite remotely stimulated by a low-frequency alternating magnetic field (LAMF)[70]. The nanocomposite was synthesized by in-situ precipitation of iron precursor to avoid the agglomeration of $\mathrm{Fe}_{3} \mathrm{O}_{4}$ nanoparticles and loss of superparamagnetic properties. Additionally, this method of nanoparticle precipitation provides high homogeneity, improving the mechanical properties of the chitosan matrix with an increase of $416 \%$ in tensile strength and $265 \%$ in elastic modulus properties. The release of heat by LAMF can increase the local temperature, modifying the diffusional coefficient of drugs, improving the release of Adriamycin and Rifampicin by around $20 \%$ (see Figure 6). 
(a)

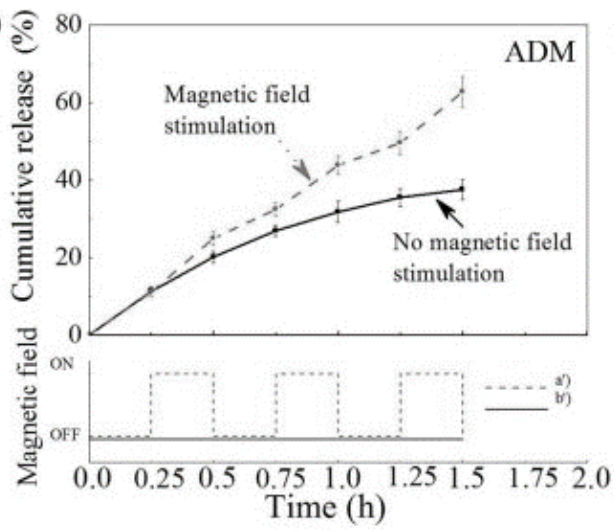

(b)

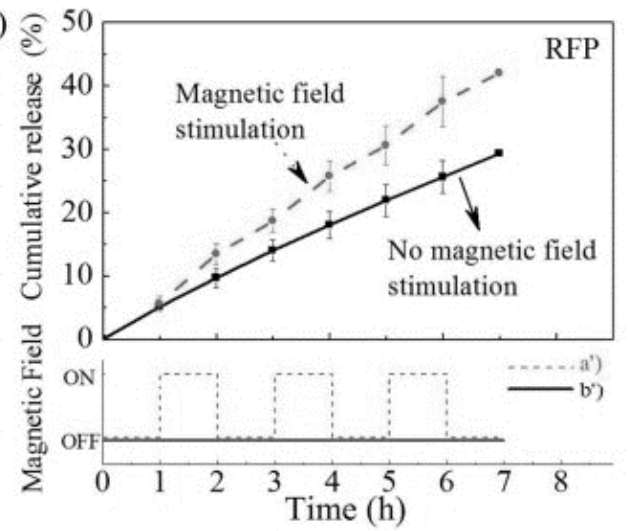

Figure 6. Comparison of cumulative drug release profile with or without LAMF pulses. a) Adriamycin and b) Rifampicin. (Adapted with permission of [70]. Copyright (2018) American Chemical Society)

Another interesting study about the release of biocomponent was developed for Zahra et al., They proposed a multicomponent material to apply in bone regeneration by osteoprogenitor cells proliferation. For this purpose, they were designed a two-compartment material to evaluate the bioactive compound release from a magneto-responsive compartment (based $\mathrm{Fe}_{3} \mathrm{O}_{4}$ and crosslinked alginate matrix loaded with bone morphogenetic protein-2) to the gel foam compartment as cell culture platform (loaded with stromal cell-derived factor 1$\alpha$ ). The deformation of the structure due to magneto-response material generate the bioactive compound release to the other compartment.

\subsection{Electric current.}

The use of electric currents is interesting in SMP or SMC materials. The application of electrical currents can produce a change in the shape of materials based on local heat release produced by the Joule heating effect. An important aspect to consider is the possibility to synthesizes composites based in a matrix with conducting properties or fillers with conducting properties. In the first case, the conducting polymers (or intrinsically conducting polymers) have been extensively studied[71-74], which must be reinforced by inactive fillers to improve the mechanical and thermal properties. Currently, the most attractive option is to usenanofillers as active components of materials. The carbon-derived nanomaterials as graphene, graphene oxide (GO) or carbon nanotubes (CNTs), stand out as nanofiller sensitive to the electrical current, due to their present excellent mechanical, thermal properties, and also improve the electronic properties significantly. However, this is limited by the randomized distribution of nanofiller in the polymer matrix[29]. Some researchers have also proposed to unite the properties of both conductive polymers, and conductive nanofillers. Yang et al., studied a polymer composed by 2-acrylamide-2-methyl propane sulfonic acid (AMPS) and acrylamide (AAm) and reduced graphene oxide (rGO) as filler, based on in-situ polymerization of GO nanoplatelets, then GO were reduced by hydrazine to obtain $\mathrm{rGO} /$ poly(AMPS-co-AAm) nanocomposite with electric controllable swelling/deswelling behavior[75]. Additionally, the mechanical properties and electrical response of nanocomposites were improved compared to the pure polymer matrix, like it is possible to see as a fast response of nanocomposite than pure polymer when it is used as a soft robot (see Figure 7).
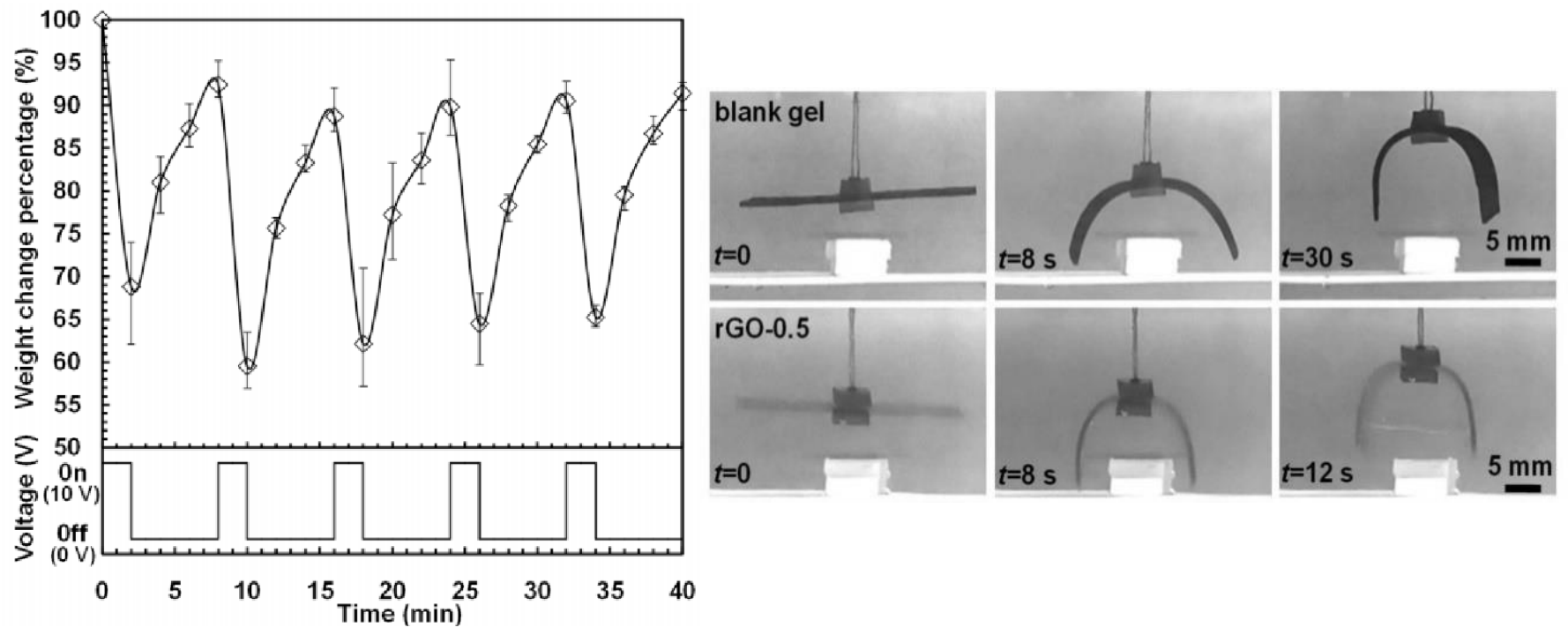

Figure 7. Magnetic response effect on rGO-0.5 nanocomposite. a) Reversible deswelling/swelling behaviors under the cyclic electric field. B)The gripping behaviors of blank gel and rGO-0.5 nanocomposite. (Adapted with permission of [75]. Copyright (2017) American Chemical Society)

Another interesting nanocomposite material was reported by Wan et al., about 4D material based on poly(D, L-lactide-co-trimethylene carbonate), where trimethylene carbonate moieties act as softness structure in the matrix, while CNTs was selected as nanofiller [76]. The nanocomposite was applied as an electronic component by testing in a simple electronic circuit, and as a liquid sensor, based on resistivity measurements produced by molecules that disrupt the normal connection between CNTs entities into the composite.
Furthermore, it is possible to modulate the contact area between the sensor device with liquid volume, improving its precision, due to the shape memory properties of the nanocomposite.

Respect to the medical application of nanocomposites, Gangrade et al., reported a single-walled carbon nanotube (SWCNTs) modified with folic acid and then loaded with the chemotherapeutic drug doxorubicin (DOX). 
The nanocomposite was synthesized by the blend of silk with drugloaded SWCNTs and then was compared their electroconductivity properties and electromagnetic response to be applied on electro/light-sensitive drug delivery system[77].

\subsection{Light.}

The use of light in different areas of science is very attractive, due to can be easily generated, or it can be used directly from solar energy. Additionally, the light is a remote-control stimulation source, and then it is not affected by electromagnetic interference compared with the electric or magnetic field. On the other hand, an important property of nanomaterials, especially metal or oxide nanoparticles, is the localized surface plasmon resonances (LSPR) by the interaction between particle surface with a light source. The LSPR is determined and finetuned by nature, shape, and size of nanomaterial[78]. This property generates intense electromagnetic fields by incident light, which then decay being able a fast heat transference from nanoparticles to the nearby environment, it is the conversion to heat release, which can produce the response of the polymer matrix, this kind of material are sometimes called plasmonic polymers composite[79]. The light-responsive nanocomposites are widely studied for medical applications, especially as drug nanocarriers and cancer treatment. Many authors have defined near-infrared (NIR) irradiation as a better option in medical applications. It has deep permeation and negligible absorbance in human tissue, at the difference to other electromagnetic irradiation as UV light[80]. Li et al. proposed a polyethyleneimine matrix modified with thiocarbamate as hydrogen sulfide $\left(\mathrm{H}_{2} \mathrm{~S}\right)$ release platform by partial decomposition activated by NIR light interaction with reduced graphene[81]. The exogenous $\mathrm{H}_{2} \mathrm{~S}$ has been studied successfully as a treatment for cancer, proving to prevent the growth of tumors[82]. Raza et al., has been widely discussed about drug delivery for cancer treatment by nanocomposites responsive to NIR irradiation. Between all work reviewed, it is possible to found improvement by modification of diffusional coefficient for different drugs or thermoresponsive polymers activated by heat release of nanoparticles responsive to NIR irradiation[83]. In the tissue regeneration area, Yang et al., proposed a nanocomposite to promote cell growth in skin lesions and, at the same time, prevent the proliferation of bacteria in the treated area[84]. The nanocomposite is based on a modified chitosan hydrogel matrix crosslinked by imine bonds with dialdehyde functionalized PEG, while tungsten disulfide nanosheets have been used as light-response nanofiller. The nanocomposites were loaded with ciprofloxacin as antibacterial treatment and were evaluated in-vivo infected system compared with control and non-infected test subject. The nanocomposite activated with NIR light effectively controlled bacterial growth and regeneration of tissue, with negligible difference compared with the non-infected subject. Yue et al studied a similar approach with interesting results., using graphene quantum dots as active NIR nanofiller in a dextran structure modified with pendant PNIPAAm chains[58]. The nanocomposite was loaded with buprenorphine as an anti-inflammatory agent, probed that thermoresponsive chains play an important role in drug release to pain control.

For SMC materials activated by light is possible to stand out the work of Cui et al., were synthesized a 4D nano-printable composite[85]. The SMP matrix is based in an epoxy monomer crosslinked by poly(propylene glycol) bis(2aminopropyl) ether present a transition temperature around $45{ }^{\circ} \mathrm{C}$, while graphene nanoplatelets used as nanofiller not varying the recovery properties, but improve the mechanical properties of SMP and the light-sensitive property. Other interesting carbonaceous SMC material, was reported by $\mathrm{Li}$ et al., the composite is based on polyvinyl alcohol (PVA) matrix. At the same time, graphene sheets grafting with polyacrylic acid (PAA) was used as nanofiller[86]. PAA grafting into graphene is closely related with great recovery shape properties of SMC because it contributes with a large amount of hydrogenbonding interaction with the PVA matrix generating a stable composite network. Additionally, the PAA also provides generating a self-healing material in a larger humidity environment. Self-healing is a desirable property in memory materials since those are subjected to constant stress that can damage the structure.

The SMC was evaluated using NIR irradiation, for the first time, a simple film of PVA/GO-PAA composite shown total shape recovery compared to a pure PVA film (Figure 8). Simultaneously, the PVA/GO-PAA preformed flower structure showed a fast recovery simulating the natural behavior of flowers (Figure 8).

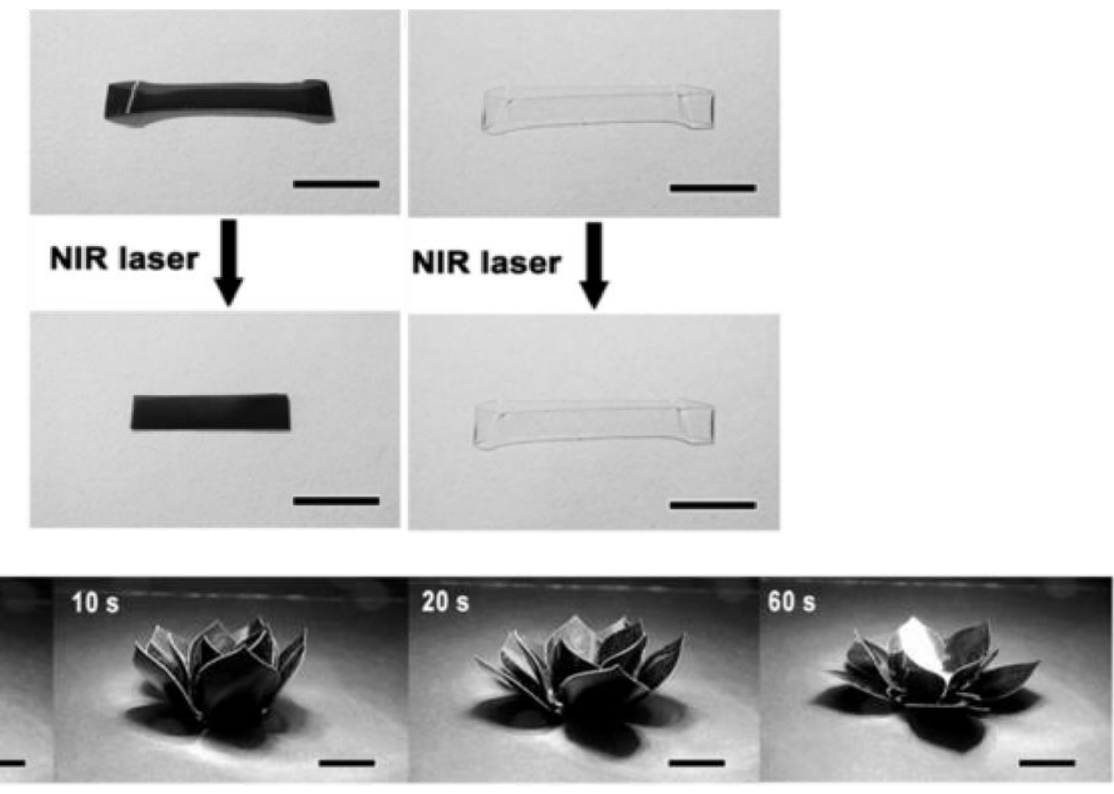

Figure 8. Shape recovery evaluation by NIR irradiation. a) Shape recovery of the PVA/PAA-GO $3 \%$ and the PVA film. b) Time-sequence images of the PVA/PAAGO $3 \%$ flower that is blooming under NIR light irradiation. (Adapted with permission of [86]. Copyright (2019) American Chemical Society).

\section{CONCLUSIONS AND OUTLOOK}

Undoubtedly nanocomposite polymers represent a niche of exciting and attractive development in the area of materials science. Furthermore, if we exploit the richness of the physical and chemical properties of its components, we can provide intelligent properties to the materials, and the potentialities are even greater. In this mini-review, we revised developments in the field of smart polymer nanocomposite to respond to external stimuli such as light, magnetic field, electric field, and temperature, emphasizing the latest biomedical applications developed in the last three years. However, there is still much to study in these systems.

One of the most challenging variables is the high surface energy of the nanofiller and, consequently, its high tendency to agglomeration, which imposes difficulties in controlling the matrix-nanofiller interface, the dispersion, and the distribution of the nanoparticles. These critical variables have a profound effect on the macroscopic properties and certainly on the responsiveness to stimuli. Until now, different methods of synthesis of nanocomposite materials as well as 
different methods for the surface modification of nanofillers aimed at obtaining a better distribution of the nanofiller in the polymer matrix have been proposed. Nevertheless, this topic still requires more attention, since due to the intrinsic properties of the nanoparticles, achieving its homogeneous dispersion in the polymer matrix remains a challenge. Studies aimed at finding strategies for the best dispersion of nanoparticles both in solutions and in the polymer matrix will bring about the development of new materials with more uniform properties and with more reproducible synthesis processes. Indeed, achieving control over all of those critical and challenging variables will give an even greater impetus to developing these materials and provide certainty about their potential for scaling. Additionally, it should be noted that this search for new synthesis and modification methodologies, both of polymeric matrices and fillers, must be closely linked to new technological solutions related to 3D printing, since there is currently a growing demand of responsive nanocomposites to obtain new technological devices by printing techniques.

\section{ACKNOWLEDGMENT}

The authors thank to FONDECYT Regular (grant $\mathrm{N}^{\circ} 1171082$ ) and ANID FONDECYT/POSTDOCTORADO/Grant N 3200379 and 3200601 .

\section{REFERENCES}

1. J.H. Koo, Polymer nanocomposites, McGraw-Hill Professional Pub.2006.

2. Y. Kojima, A. Usuki, M. Kawasumi, A. Okada, Y. Fukushima, T. Kurauchi, O. Kamigaito, Mechanical properties of nylon 6-clay hybrid, Journal of Materials Research. 8, 1185-1189, (1993).

3. S. Fu, Z. Sun, P. Huang, Y. Li, N. Hu, Some basic aspects of polymer nanocomposites: A critical review, Nano Materials Science. 1, 2-30, (2019).

4. D. Ponnamma, J.-J. Cabibihan, M. Rajan, S.S. Pethaiah, K. Deshmukh, J.P. Gogoi, S.K. Pasha, M.B. Ahamed, J. Krishnegowda, B. Chandrashekar, Synthesis, optimization and applications of $\mathrm{ZnO} /$ polymer nanocomposites, Materials Science and Engineering: C. 98, 1210-1240, (2019) .

5. L. Hsu, C. Weder, S.J. Rowan, Stimuli-responsive, mechanically-adaptive polymer nanocomposites, Journal of Materials Chemistry. 21, 2812-2822, (2011)

6. K.I. Winey, R.A. Vaia, Polymer nanocomposites, MRS bulletin. 32, 314-322, (2007)

7. W. Chow, Z.M. Ishak, Smart polymer nanocomposites: A review, Express Polymer Letters. 14, 416-435, (2020).

8. J. Leng, A.K.-t. Lau, Multifunctional polymer nanocomposites, CRC press 2010.

9. A. Pourjavadi, S. Rahemipoor, M. Kohestanian, Synthesis and characterization of multi stimuliresponsive block copolymer-silica hybrid nanocomposite with core-shell structure via RAFT polymerization, Composites Science and Technology. 188, 107951, (2020).

10. A. Seyfoori, S. Sarfarazijami, S. Seyyed Ebrahimi, pH-responsive carbon nanotube-based hybrid nanogels as the smart anticancer drug carrier, Artificial Cells, Nanomedicine, and Biotechnology. 47, 14371443, (2019).

11. W. Wu, Stretchable electronics: functional materials, fabrication strategies and applications, Science and Technology of Advanced Materials. 20, 187224, (2019).

12. M. Bashir, P. Rajendran, A review on electroactive polymers development for aerospace applications, Journal of Intelligent Material Systems and Structures. 29, 3681-3695, (2018)

13. D. Feldman, Polymers and polymer nanocomposites for cancer therapy, Applied Sciences. 9, 3899, (2019)

14. M. Malaki, W. Xu, A.K. Kasar, P.L. Menezes, H. Dieringa, R.S. Varma, M. Gupta, Advanced metal matrix nanocomposites, Metals. 9, 330, (2019).

15. S. Bull, Multifunctional polymer nanocomposites for industrial applications, Nanofibers and Nanotechnology in Textiles, Elsevier 2007, pp. 256-280.

16. E. Kazemi-Khasragh, F. Bahari-Sambran, C. Platzer, R. Eslami-Farsani, The synergistic effect of graphene nanoplatelets-montmorillonite hybrid system on tribological behavior of epoxy-based nanocomposites, Tribology International. 106472, (2020).

17. K.K. Khichar, S.B. Dangi, V. Dhayal, U. Kumar, S.Z. Hashmi, V. Sadhu, B.L. Choudhary, S. Kumar, S. Kaya, A.E. Kuznetsov, Structural, optical, and surface morphological studies of ethyl cellulose/graphene oxide nanocomposites, Polymer Composite, 41, 2792-2802, (2020).
18. C. Miao, W.Y. Hamad, Critical insights into the reinforcement potential of cellulose nanocrystals in polymer nanocomposites, Current Opinion in Solid State and Materials Science. 23, 100761, (2019)

19. T.T. Zhu, C.H. Zhou, F.B. Kabwe, Q.Q. Wu, C.S. Li, J.R. Zhang, Exfoliation of montmorillonite and related properties of clay/polymer nanocomposites, Applied Clay Science. 169, 48-66, (2019).

20. J. Yin, F. Zhan, T. Jiao, H. Deng, G. Zou, Z. Bai, Q. Zhang, Q. Peng, Highly efficient catalytic performances of nitro compounds via hierarchical PdNPsloaded MXene/polymer nanocomposites synthesized through electrospinning strategy for wastewater treatment, Chinese Chemical Letters. 31, 992-995, (2020).

21. E. Akpan, X. Shen, B. Wetzel, K. Friedrich, Design and synthesis of polymer nanocomposites, Polymer Composites with Functionalized Nanoparticles, Elsevier 2019, pp. 47-83.

22. B. Ballarin, E. Boanini, L. Montalto, P. Mengucci, D. Nanni, C. Parise, I Ragazzini, D. Rinaldi, N. Sangiorgi, A. Sanson, PANI/Au/Fe3O4 Nanocomposite materials for high performance energy storage, Electrochimica Acta. 322, 134707, (2019).

23. A. Kumar, K. Sharma, A.R. Dixit, A review of the mechanical and thermal properties of graphene and its hybrid polymer nanocomposites for structural applications, Journal of materials science. 54, 59926026, (2019).

24. T. Liu, T. Zhou, Y. Yao, F. Zhang, L. Liu, Y. Liu, J. Leng, Stimulus methods of multi-functional shape memory polymer nanocomposites: A review, Composites Part A: Applied Science and Manufacturing. 100, 20-30, (2017)

25. N. Olivera, T.B. Rouf, J.C. Bonilla, J.G. Carriazo, N. Dianda, J.L. Kokini, Effect of LAPONITE® addition on the mechanical, barrier and surface properties of novel biodegradable kafirin nanocomposite films, Journal of Food Engineering. 245, 24-32, (2019).

26. M. Albdiry, B. Yousif, Toughening of brittle polyester with functionalized halloysite nanocomposites, Composites Part B: Engineering. 160, 94-109, (2019).

27. Y. Lin, S. Hu, G. Wu, Structure, Dynamics, and Mechanical Properties of Polyimide-Grafted Silica Nanocomposites, The Journal of Physical Chemistry C. 123, 6616-6626, (2019).

28. G. Pang, S. Zhang, X. Zhou, H. Yu, Y. Wu, T. Jiang, X. Zhang, F. Wang, Y Wang, L.W. Zhang, Immunoactive polysaccharide functionalized gold nanocomposites promote dendritic cell stimulation and antitumor effects, Nanomedicine. 14, 1291-1306, (2019).

29. Q. Lu, H.S. Jang, W.J. Han, J.H. Lee, H.J. Choi, Stimuli-responsive graphene oxide-polymer nanocomposites, Macromolecular Research. 27, 1061-1070, (2019).

30. D. Ponnamma, H. Parangusan, A. Tanvir, M.A.A. AlMa'adeed, Smart and robust electrospun fabrics of piezoelectric polymer nanocomposite for selfpowering electronic textiles, Materials \& Design. 184, 108176, (2019).

31.Z. Zarnegar, J. Safari, Z. Zahraei, Design, synthesis and antimicrobial evaluation of silver decorated magnetic polymeric nanocomposites, NanoStructures \& Nano-Objects. 19, 100368, (2019).

32. M. Li, Y. Shi, H. Gao, Z. Chen, Bio-Inspired Nanospiky Metal Particles Enable Thin, Flexible, and Thermo-Responsive Polymer Nanocomposites for Thermal Regulation, Advanced Functional Materials. 30, 1910328, (2020).

33. Y. Chen, X. Zhao, C. Luo, M.-B. Yang, B. Yin, A facile fabrication of shape memory polymer nanocomposites with fast light-response and self-healing performance, Composites Part A: Applied Science and Manufacturing. 105931, (2020).

34. Y. Zhang, K. Chen, Y. Li, J. Lan, B. Yan, L. Shi, R. Ran, High-strength, selfhealable, temperaturesensitive, MXene-containing composite hydrogel as a smart compression sensor, ACS Applied Materials \& Interfaces. 11, 4735047357, (2019).

35. R.-Q. Zhang, L.-B. Wang, R.-X. Bai, Y.-L. Luo, F. Xu, Y.-S. Chen, Sensitive conductive polymer nanocomposites from multiwalled carbon nanotube coated with polypyrrole and hydroxyl-terminated poly (butadiene-coacrylonitile) polyurethane for detection of chloroform vapor, Composites Part B: Engineering. 173, 106894, (2019).

36. S. Li, Y. Zhang, Q. You, Q. Wang, G. Liao, D. Wang, Highly efficient removal of antibiotics and dyes from water by the modified carbon nanofibers composites with abundant mesoporous structure, Colloids and Surfaces A: Physicochemical and Engineering Aspects. 558, 392-401, (2018).

37. C. Wang, L. Ma, Q. Wen, B. Wang, R. Han, C. Hao, K. Chen, Enhanced electrorheological characteristics of titanium oxide@ H2Ti2O5 nanotube 
core/shell nanocomposite, Colloids and Surfaces A: Physicochemical and Engineering Aspects. 578, 123641, (2019).

38. M. Pastucha, Z. Farka, K. Lacina, Z. Mikušová, P. Skládal, Magnetic nanoparticles for smart electrochemical immunoassays: a review on recent developments, Microchimica Acta. 186, 312, (2019).

39. R. Kurahatti, A. Surendranathan, S. Kori, N. Singh, A. Kumar, S. Srivastava, Defence applications of polymer nanocomposites, Defence Science Journal, 60, 551-563, (2010).

40. M. Rong, M. Zhang, W. Ruan, Surface modification of nanoscale fillers for improving properties of polymer nanocomposites: a review, Materials science and technology. 22, 787-796, (2006).

41. Y. Ou, F. Yang, Z.Z. Yu, A new conception on the toughness of nylon 6/silica nanocomposite prepared via in situ polymerization, Journal of Polymer Science Part B: Polymer Physics. 36, 789-795, (1998).

42. C. Harito, D.V. Bavykin, B. Yuliarto, H.K. Dipojono, F.C. Walsh, Polymer nanocomposites having a high filler content: synthesis, structures, properties, and applications, Nanoscale. 11, 4653-4682, (2019).

43. B. Wang, D. Peng, R. Lv, B. Na, H. Liu, Z. Yu, Generic melt compounding strategy using reactive graphene towards high performance polyethylene/graphene nanocomposites, Composites Science and Technology. 177, 1-9, (2019).

44. X. Wu, S. Takeshita, K. Tadumi, W. Dong, S. Horiuchi, H. Niino, T. Furuya, S. Yoda, Preparation of noble metal/polymer nanocomposites via in situ polymerization and metal complex reduction, Materials Chemistry and Physics. 222, 300-308, (2019).

45. M. Herrero, M. Asensio, K. Núñez, J.C. Merino, J.M. Pastor, Morphological, Thermal, and Mechanical Behavior of Polyamide11/Sepiolite BioNanocomposites Prepared by Melt Compounding and In Situ Polymerization, Polymer Composites. 40, E704-E713, (2019).

46. I. Bayraktar, D. Doganay, S. Coskun, C. Kaynak, G. Akca, H.E. Unalan, 3D printed antibacterial silver nanowire/polylactide nanocomposites, Composites Part B: Engineering. 172, 671-678, (2019).

47. V.A. Tanna, J.S. Enokida, E.B. Coughlin, H.H. Winter, Functionalized Polybutadiene for Clay-Polymer Nanocomposite Fabrication, Macromolecules. 52, 6135-6141, (2019).

48. J. Jordan, K.I. Jacob, R. Tannenbaum, M.A. Sharaf, I. Jasiuk, Experimental trends in polymer nanocomposites - a review, Materials science and engineering: A. 393, 1-11, (2005).

49. H. Behniafar, A. Ahmadi-khaneghah, M. Yazdi, Enhanced heat stability and storage modulus in novel PTMO-intercalated clay platelets/PTMO-based polyurethane nanocomposites, Journal of Polymer Research. 23, 202, (2016)

50. V. S Wadi, K.K. Jena, K. Halique, S.M. Alhassan, Enhanced Mechanical Toughness of Isotactic Polypropylene Using Bulk Molybdenum Disulfide, ACS Omega , 5, 11394-11401 (2020).

51. L. Toledo, D. Palacio, S. Sánchez, B.F. Urbano, Pluronic-coated nanoparticles for enhanced spatial distribution and increased softness of nanocomposite hydrogels, Journal of Materials Science. 55, 89688982 , (2020).

52. L. Toledo, D.A. Palacio, B.F. Urbano, Tuning the softness of poly(2hydroxyethyl methacrylate) nanocomposite hydrogels through the addition of PEG coated nanoparticles, Journal of Colloid and Interface Science. 578, 749-757, (2020)

53. J. Cha, J. Kim, S. Ryu, S.H. Hong, Comparison to mechanical properties of epoxy nanocomposites reinforced by functionalized carbon nanotubes and graphene nanoplatelets, Composites Part B: Engineering. 162, 283-288, (2019).

54. C. Min, D. Liu, J. Qian, Z. He, W. Jia, H. Song, L. Guo, High mechanical and tribological performance polyimide nanocomposites using aminefunctionalized graphene nanosheets, Tribology International. 131, 1-10, (2019).

55. J. Niskanen, H. Tenhu, How to manipulate the upper critical solution temperature (UCST)?, Polymer Chemistry. 8, 220-232, (2017)

56. Q. Zhang, R. Hoogenboom, Polymers with upper critical solution temperature behavior in alcohol/water solvent mixtures, Progress in Polymer Science. 48, 122-142, (2015).

57. R. Hoogenboom, Chapter 2 - Temperature-Responsive Polymers: Properties, Synthesis, and Applications, in: M.R. Aguilar, J. San Román (Eds.), Smart Polymers and their Applications (Second Edition), Woodhead Publishing 2019, pp. 13-44.
58. J. Yue, L. He, Y. Tang, L. Yang, B. Wu, J. Ni, Facile design and development of photoluminescent graphene quantum dots grafted dextran/glycolpolymeric hydrogel for thermoresponsive triggered delivery of buprenorphine on pain management in tissue implantation, Journal of Photochemistry and Photobiology B: Biology. 197, 111530, (2019).

59. N.M. Tatiana, V. Cornelia, R. Tatia, C. Aurica, Hybrid collagen/pNIPAAM hydrogel nanocomposites for tissue engineering application, Colloid and Polymer Science. 296, 1555-1571, (2018).

60. Ö. Demir Oğuz, D. Ege, Rheological and Mechanical Properties of Thermoresponsive Methylcellulose/Calcium Phosphate-Based Injectable Bone Substitutes, Materials. 11, 604, (2018).

61. M.G. Arafa, R.F. El-Kased, M.M. Elmazar, Thermoresponsive gels containing gold nanoparticles as smart antibacterial and wound healing agents, Scientific Reports. 8, 13674, (2018).

62. L. Li, R. Niu, Y. Zhang, Ag-Au bimetallic nanocomposites stabilized with organic-inorganic hybrid microgels: synthesis and their regulated optical and catalytic properties, RSC Advances. 8, 12428-12438, (2018).

63. Q. Feng, D. Tang, H. Lv, W. Zhang, W. Li, Surface-initiated ATRP to modify $\mathrm{ZnO}$ nanoparticles with poly(N-isopropylacrylamide): Temperaturecontrolled switching of photocatalysis, Journal of Alloys and Compounds. 691, 185-194, (2017).

64. S. Van Berkum, T.J. Dee, P.A. Philipse, H.B. Erné, Frequency-Dependent Magnetic Susceptibility of Magnetite and Cobalt Ferrite Nanoparticles Embedded in PAA Hydrogel, International Journal of Molecular Sciences. 14, 10162-10177, (2013)

65. J. Kudr, Y. Haddad, L. Richtera, Z. Heger, M. Cernak, V. Adam, O. Zitka Magnetic Nanoparticles: From Design and Synthesis to Real World Applications, Nanomaterials (Basel, Switzerland). 7, 243, (2017).

66. H. Yang, W.R. Leow, T. Wang, J. Wang, J. Yu, K. He, D. Qi, C. Wan, X Chen, 3D Printed Photoresponsive Devices Based on Shape Memory Composites, Advanced Materials. 29, 1701627, (2017).

67. G.D. Soto, C. Meiorin, D.G. Actis, P. Mendoza Zélis, O. Moscoso Londoño, D. Muraca, M.A. Mosiewicki, N.E. Marcovich, Magnetic nanocomposites based on shape memory polyurethanes, European Polymer Journal. 109, 815, (2018).

68. W. Zhao, F. Zhang, J. Leng, Y. Liu, Personalized 4D printing of bioinspired tracheal scaffold concept based on magnetic stimulated shape memory composites, Composites Science and Technology. 184, 107866, (2019).

69. H. Wei, Q. Zhang, Y. Yao, L. Liu, Y. Liu, J. Leng, Direct-Write Fabrication of 4D Active Shape-Changing Structures Based on a Shape Memory Polymer and Its Nanocomposite, ACS Applied Materials \& Interfaces. 9, 876-883, (2017).

70. Y. Wang, B. Li, F. Xu, Z. Han, D. Wei, D. Jia, Y. Zhou, Tough Magnetic Chitosan Hydrogel Nanocomposites for Remotely Stimulated Drug Release, Biomacromolecules. 19, 3351-3360, (2018).

71. P.P. Deshpande, N.G. Jadhav, V.J. Gelling, D. Sazou, Conducting polymers for corrosion protection: a review, Journal of Coatings Technology and Research. 11, 473-494, (2014).

72. M. Ates, A review study of (bio)sensor systems based on conducting polymers, Materials Science and Engineering: C. 33, 1853-1859, (2013).

73. T.K. Das, S. Prusty, Review on Conducting Polymers and Their Applications, Polymer-Plastics Technology and Engineering. 51, 1487-1500, (2012).

74. D.W. Hatchett, M. Josowicz, Composites of Intrinsically Conducting Polymers as Sensing Nanomaterials, Chemical Reviews. 108, 746-769, (2008).

75. C. Yang, Z. Liu, C. Chen, K. Shi, L. Zhang, X.-J. Ju, W. Wang, R. Xie, L.Y. Chu, Reduced Graphene Oxide-Containing Smart Hydrogels with Excellent Electro-Response and Mechanical Properties for Soft Actuators, ACS Applied Materials \& Interfaces. 9, 15758-15767, (2017).

76. X. Wan, F. Zhang, Y. Liu, J. Leng, CNT-based electro-responsive shape memory functionalized 3D printed nanocomposites for liquid sensors, Carbon. 155, 77-87, (2019)

77. A. Gangrade, B. Gawali, P.K. Jadi, V.G.M. Naidu, B.B. Mandal, PhotoElectro Active Nanocomposite Silk Hydrogel for Spatiotemporal Controlled Release of Chemotherapeutics: An In Vivo Approach toward Suppressing Solid Tumor Growth, ACS Applied Materials \& Interfaces. 12, 27905-27916, (2020).

78. J. Zeng, W. Duan, M. Li, Y. Xue, Chapter 11 - Plasmonic Metallic Nanostructures as Colorimetric Probes for Environmental Pollutants, in: X. 
Wang, X. Chen (Eds.), Novel Nanomaterials for Biomedical, Environmental and Energy Applications, Elsevier 2019, pp. 327-352.

79. I. Pastoriza-Santos, C. Kinnear, J. Pérez-Juste, P. Mulvaney, L.M. LizMarzán, Plasmonic polymer nanocomposites, Nature Reviews Materials. 3, 375-391, (2018).

80. C.-H. Zhu, Y. Lu, J. Peng, J.-F. Chen, S.-H. Yu, Photothermally Sensitive Poly(Nisopropylacrylamide)/Graphene Oxide Nanocomposite Hydrogels as Remote Light-Controlled Liquid Microvalves, Advanced Functional Materials. 22, 4017-4022, (2012).

81. H. Li, Y. Yao, H. Shi, Y. Lei, Y. Huang, K. Wang, X. He, J. Liu, A nearinfrared light-responsive nanocomposite for photothermal release of $\mathrm{H} 2 \mathrm{~S}$ and suppression of cell viability, Journal of Materials Chemistry B. 7, 5992-5997, (2019).

82. A Review of Hydrogen Sulfide Synthesis, Metabolism, and Measurement: Is Modulation of Hydrogen Sulfide a Novel Therapeutic for Cancer? Antioxidants \& Redox Signaling. 31, 1-38, (2019).
83. A. Raza, U. Hayat, T. Rasheed, M. Bilal, H.M.N. Iqbal, "Smart” materialsbased near-infrared lightresponsive drug delivery systems for cancer treatment: A review, Journal of Materials Research and Technology. 8, 1497 $1509,(2019)$

84. N. Yang, M. Zhu, G. Xu, N. Liu, C. Yu, A near-infrared light-responsive multifunctional nanocomposite hydrogel for efficient and synergistic antibacterial wound therapy and healing promotion, Journal of Materials Chemistry B. 8, 3908-3917, (2020).

85. H. Cui, S. Miao, T. Esworthy, S.-j. Lee, X. Zhou, S.Y. Hann, T.J. Webster, B.T. Harris, L.G. Zhang, A novel near-infrared light responsive 4D printed nanoarchitecture with dynamically and remotely controllable transformation, Nano Research. 12, 1381-1388, (2019).

86. T. Li, Y. Li, X. Wang, X. Li, J. Sun, Thermally and Near-Infrared LightInduced Shape Memory Polymers Capable of Healing Mechanical Damage and Fatigued Shape Memory Function, ACS Applied Materials \& Interfaces. 11, 9470-9477, (2019). 